\title{
Erratum to: Comparative assessment of surgeons' task performance and surgical ergonomics associated with conventional and modified flank positions: a simulation study
}

\author{
Yu Fan • Gaiqing Kong • Yisen Meng • \\ Shutao Tan · Kunlin Wei $\cdot$ Qian Zhang • \\ Jie Jin
}

Published online: 15 January 2015

(c) Springer Science+Business Media New York 2015

Erratum to: Surg Endosc (2014) 28:3249-3256

DOI 10.1007/s00464-014-3598-3

The following Acknowledgment was inadvertently omitted:

\begin{abstract}
Acknowledgments Thanks to Dr. Sophia Dai from Harvard University for suggestions on study design and linguistic modification. The study was supported by the National Natural Science Foundation of China (Grant Nos. 31000456, 31371020, J1103602, and 61020106005), the Fundamental Research Funds for the Central Universities, National High Technology Research and Development Program of China (863 Program, Grant 2012AA011602).
\end{abstract}

The online version of the original article can be found under doi: 10 . 1007/s00464-014-3598-3.

Y. Fan $(\bowtie) \cdot$ Y. Meng $\cdot$ Q. Zhang $(\bowtie) \cdot$ J. Jin

Urology Department and Institute of Urology, Peking University

First Hospital, Peking University, Beijing 100034, China

e-mail: dantefanbmu@126.com

Q. Zhang

e-mail: zhangqianbmu@vip.sina.com

G. Kong · K. Wei $(\bowtie)$

Department of Psychology, Peking University, Beijing 100080,

China

e-mail: wei_kunlin@126.com

S. Tan

Shengjing Hospital of China Medical University, Shenyang,

China 